\title{
ALGEBRAIC ANALYSIS OF MANY VALUED LOGICS $\left({ }^{1}\right)$
}

\author{
BY \\ C. C. CHANG
}

This paper is an attempt at developing a theory of algebraic systems that would correspond in a natural fashion to the $\boldsymbol{\aleph}_{0}$-valued propositional calculus $\left({ }^{2}\right)$. For want of a better name, we shall call these algebraic systems $\mathrm{MV}$-algebras where $\mathrm{MV}$ is supposed to suggest many-valued logics. It is known that the classical two-valued logic gives rise to the study of Boolean algebras and, as can be expected, every Boolean algebra will be an MValgebra whereas the converse does not hold. However, many results for Boolean algebras can be appropriately carried over to MV-algebras, although in some cases the proofs become more subtle and delicate. The motivation behind the present study is to find a proof of the completeness of the $\boldsymbol{N}_{0}$ valued logic by using some algebraic results concerning $\mathrm{MV}$-algebras; more specifically, it is known that the completeness of the two-valued logic is a consequence of the Boolean prime ideal theorem and we wish to exploit just some such corresponding result for $\mathrm{MV}$-algebras $\left({ }^{3}\right)$. It will be seen that our effort in duplicating this result is only partially successful. In the first four sections of this paper we present various theorems concerning both the arithmetic in $\mathrm{MV}$-algebras and the structure of these algebras. In the last section we give some applications of our results to the study of completeness of $\boldsymbol{\aleph}_{0}$-valued logic and some related topics. We point out here that the treatment of MV-algebras as given here is not meant to be complete and exhaustive.

1. Axioms of MV-algebras and some elementary consequences. An MValgebra is a system $\langle A,+, \cdot,-, 0,1\rangle$ where $A$ is a nonempty set of elements, 0 and 1 are distinct constant elements of $A,+$ and $\cdot$ are binary operations on elements of $A$, and - is a unary operation on elements of $A$ obeying the following axioms. (We assume here, of course, that $A$ is closed under the operations,$+ \cdot$, and -.)

Received by the editors January 8, 1957.

(1) The research and preparation of this paper were supported in part by the United States Navy under Contract No. NONR 401 (20)-NR 043-167 monitored by the Office of Naval Research; reproduction in whole or in part is permitted for any purpose of the United States Government.

(2) We have in mind specifically the $\boldsymbol{N}_{0}$-valued propositional calculus developed in [3] and [5]; the axioms for this calculus are known as the Eukasiewicz axioms.

$\left.{ }^{3}\right)$ It is of course known that the completeness of the Eukasiewicz axioms for $\boldsymbol{\aleph}_{0}$-valued logic has been proved in [5]. This result was also stated on p. 240 in [8]; however, it was never published. 
Ax. 1. $x+y=y+x$.

Ax. 2. $x+(y+z)=(x+y)+z$.

Ax. 3. $x+\bar{x}=1$.

Ax. 4. $x+1=1$.

Ax. 5. $x+0=x$.

Ax. 6. $[x+y]-=\bar{x} \cdot \bar{y}$.

Ax. 7. $x=[\bar{x}]-$.
Ax. 1'. $x \cdot y=y \cdot x$.

Ax. $2^{\prime} \cdot x \cdot(y \cdot z)=(x \cdot y) \cdot z$.

Ax. $3^{\prime} \cdot x \cdot \bar{x}=0$.

Ax. $4^{\prime} \cdot x \cdot 0=0$.

Ax. $5^{\prime} \cdot x \cdot 1=x$.

Ax. 6'. $[x \cdot y]^{-}=\bar{x}+\bar{y}$.

Ax. 8. $\overline{0}=1$.

In order to write the remaining axioms in a compact form we introduce the following definition.

DEFINITION 1.1. $x \bigvee y=(x \cdot \bar{y})+y$.

$$
x \wedge y=(x+\bar{y}) \cdot y \text {. }
$$

Ax. 9. $x \vee y=y \vee x$.

Ax. 9'. $x \wedge y=y \wedge x$.

Ax. 10. $x \bigvee(y \bigvee z)=(x \bigvee y) \bigvee z$

Ax. $10^{\prime} . x \wedge(y \wedge z)=(x \wedge y) \wedge z$.

Ax. 11. $x+(y \wedge z)=(x+y) \wedge(x+z)$.

Ax. $11^{\prime} . x \cdot(y \bigvee z)=(x \cdot y) \bigvee(x \cdot z)$

It is clear that this axiom system is not the most economical one; they are given in the above form for their intuitive contents. It is also clear that, just as in the case of Boolean algebras, there is a duality involving the elements 0 and 1 , the operations + and $\cdot$, and the operations $\vee$ and $\wedge$. Thus any theorem stated will have as an easy consequence from the axioms its dual. We make the convention, as in the case of ordinary arithmetic, that - shall be more binding than + , and, by the associative laws, we shall omit the usual parentheses in expressions of the form $x+(y+z)$ etc. Since the meanings of the axioms are clear we shall usually use them without mentioning them specifically; this is especially true for the first ten axioms. In the following theorems whenever the variables $x, y, z, \cdots$ occur they are assumed to be the elements of some fixed MV-algebra $A$.

Theorem 1.2. (i) $x \vee 0=x=x \wedge 1, x \wedge 0=0$, and $x \bigvee 1=1$.

(ii) $x \vee x=x=x \wedge x$.

(iii) $[x \vee y]^{-}=\bar{x} \wedge \bar{y}$ and $[x \wedge y]^{-}=\bar{x} \vee \bar{y}$.

(iv) $x \wedge(x \vee y)=x=x \vee(x \wedge y)$

(v) If $x+y=0$, then $x=y=0$.

(vi) If $x \cdot y=1$, then $x=y=1$.

(vii) If $x \bigvee y=0$, then $x=y=0$.

(viii) If $x \wedge y=1$, then $x=y=1$.

Proof. (i)-(iii) are obvious from 1.1 and the axioms. By the same token $x \wedge(x \bigvee y)=(y \bigvee x) \wedge x=(y \cdot \bar{x}+x) \wedge x=(y \cdot \bar{x}+x+\bar{x}) \cdot x=(y \cdot \bar{x}+1) \cdot x=1 \cdot x=x$. The other equality of (iv) follows from duality. If $x+y=0$, then $0=x \wedge 0$ $=x \wedge(x+y)=(x+0) \wedge(x+y)=x+(0 \wedge y)=x+0=x$. Thus, also $y=0$. (vi) follows from (v) by duality. If $x \vee y=0$, then by $1.1,(x \cdot \bar{y})+y=0$ hence by (v) $y=0$ and also $x=0$. (viii) again follows from (vii) by duality.

We introduce an inclusion relation $\leqq$ among the elements of an MValgebra: 
Definition 1.3. $x \leqq y$ if, and only if, $x \vee y=y$. As usual the notation $x<y$ shall mean $x \leqq y$ and $x \neq y$.

THEOREM 1.4 .

(i) $0 \leqq x \leqq 1$.

(ii) $x \leqq x$.

(iii) If $x \leqq y$ and $y \leqq z$, then $x \leqq z$.

(iv) If $x \leqq y$ and $y \leqq x$, then $x=y$.

(v) $x \leqq y$ if and only if $x \wedge y=x$.

(vi) $x \leqq y$ if and only if $\bar{y} \leqq \bar{x}$.

Proof. (i)-(iv) are obvious by 1.1-1.3 and the axioms. (v) follows immediately from 1.2 (iv), and (vi) follows from (v).

Theorem 1.5 (Monotony of $\vee$ AND $\wedge$ ). If $x \leqq y$, then $x \vee z \leqq y \vee z$ and $x \wedge z \leqq y \wedge z$.

Proof. If $x \leqq y$, then by 1.1 and $1.4(\mathrm{v}), x \wedge y=x$ and $x \vee y=y$. Thus $(x \vee z) \bigvee(y \vee z)=x \vee y \vee z \vee z=y \vee z$ and $(x \wedge z) \wedge(y \wedge z)=x \wedge y \wedge z \wedge z=x \wedge z$. This implies $x \bigvee z \leqq y \vee z$ and $x \wedge z \leqq y \wedge z$ by 1.1 and 1.4(v).

THEOREM 1.6. $x \wedge y \leqq x \leqq x \vee y$.

Proof. By 1.2(i), 1.4(i), and 1.5.

Theorem 1.7. If $x \leqq y$ and $x^{\prime} \leqq y^{\prime}$, then $x \bigvee x^{\prime} \leqq y \vee y^{\prime}$ and $x \wedge x^{\prime} \leqq y \wedge y^{\prime}$.

Proof. By 1.5, $x \vee x^{\prime} \leqq x \vee y^{\prime} \leqq y \vee y^{\prime}$. Similarly $x \wedge x^{\prime} \leqq x \wedge y^{\prime} \leqq y \wedge y^{\prime}$.

Theorem 1.8 (Monotony of + AND ·). If $x \leqq y$, then $x+z \leqq y+z$ and $x \cdot z \leqq y \cdot z$.

Proof. We note again that if $x \leqq y$ then $x \wedge y=x$ and $x \vee y=y$. By Ax. 11, $(x+z) \wedge(y+z)=(x \wedge y)+z=x+z$. Hence $x+z \leqq y+z$. Similarly, by Ax. 11', $x \cdot z \leqq y \cdot z$.

THEOREM 1.9. $x \cdot y \leqq x \leqq x+y$.

Proof. By Ax. 5, Ax. 5', 1.4(i), and 1.8.

Theorem 1.10. If $x \leqq y$ and $x^{\prime} \leqq y^{\prime}$, then $x+x^{\prime} \leqq y+y^{\prime}$ and $x \cdot x^{\prime} \leqq y \cdot y^{\prime}$.

Proof. By 1.8 .

THEOREM 1.11. The relation $\leqq$ is a partial ordering relation among the elements of $A$. The elements $x \bigvee y$ and $x \wedge y$ are respectively the l.u.b. and the g.l.b. of the elements $x$ and $y$ with respect to the ordering $\leqq$.

Proof. By 1.4, 1.6, and 1.7.

Putting 1.6, 1.9, and 1.11 together, we obtain

THEOREM 1.12. $x \cdot y \leqq x \wedge y \leqq x \leqq x \vee y \leqq x+y$. 
The next theorem will be very useful.

THEOREM 1.13. The following conditions are equivalent:

(i) $x \leqq y$, (ii) $y+\bar{x}=1$, and (iii) $x \cdot \bar{y}=0$.

Proof. Clearly (ii) and (iii) are equivalent. If $x \cdot \bar{y}=0$, then by $1.1, x \vee y$ $=x \cdot \bar{y}+y=0+y=y$ and hence $x \leqq y$. If $x \leqq y$, by $1.10,1=x+\bar{x} \leqq y+\bar{x}$. But $y+\bar{x} \leqq 1$, hence $y+\bar{x}=1$.

We have the following interesting cancellation law.

Theorem 1.14. If $x+z=y+z, x \leqq \bar{z}$, and $y \leqq \bar{z}$, then $x=y$.

Proof. By Ax. 9', $x=x \cdot 1=x \cdot(\bar{x}+\bar{z})=\bar{z} \cdot(x+z)=\bar{z} \cdot(y+z)=y \cdot(\bar{y}+\bar{z})=y \cdot 1$ $=y$.

The following theorem is motivated by $1.4(\mathrm{v})$ and 1.13 .

THEOREM 1.15. The following four conditions are equivalent:

(i) $x+y=y$.

(ii) $x \cdot y=x$.

(iii) $y \bigvee \bar{x}=1$

(iv) $x \wedge \bar{y}=0$.

Proof. We shall prove the equivalence of (ii) and (iii). Then by duality the equivalence of (i) and (iv) will follow. Clearly (iii) is equivalent with (iv). If $x \cdot y=x$, then $\bar{x} \vee y=\bar{x}+(x \cdot y)=\bar{x}+x=1$. If $\bar{x} \vee y=1$, then by Ax. 11', $x=x \cdot 1=x \cdot(\bar{x} \bigvee y)=x \cdot \bar{x} \bigvee x \cdot y=0 \bigvee x \cdot y=x \cdot y$.

THEOREM 1.16. The following conditions are equivalent:

(i) $x+x=x$.

(ii) $x \cdot x=x$.

(iii) $\bar{x}+\bar{x}=\bar{x}$.

(iv) $\bar{x} \cdot \bar{x}=\bar{x}$.

(v) $x \vee \bar{x}=1$

(vi) $x \wedge \bar{x}=0$.

Proof. By 1.15 .

1.16 points out the interesting fact that in an MV-algebra the set of elements $B$ which are idempotent with respect to the operations + or · are precisely those elements which satisfy the law of the excluded middle with respect to the operations $\vee$ or $\wedge$. Furthermore, 1.16 leads to the following

Theorem 1.17. Let $B$ be the set of elements $x$ of $A$ such that $x+x=x$. Then $B$ is closed under the operations,$+ \cdot$, and - and where $x+y=x \vee y$ and $x \cdot y$ $=x \wedge y$ for $x, y \in B$. Furthermore, the system $\langle B,+, \cdot,-, 0,1\rangle$ is not only a subalgebra of $A$ but is also the largest subalgebra of $A$ which is at the same time a Boolean algebra with respect to the same operations,$+ \cdot$, and -.

Proof. The fact that $B$ is closed under the three operations follows im- 
mediately from 1.16. Clearly $B$ is also closed under the operations $\vee$ and $\wedge$. Now, in order to prove that $B$ is a Boolean algebra we notice that if $x, y \in B$ then, since $x \leqq x \bigvee y \in B$ and $y \leqq x \vee y \in B, \quad x+y \leqq(x \vee y)+(x \bigvee y)=x \bigvee y$. But by $1.12 x \vee y \leqq x+y$. Therefore $x+y=x \vee y$ and, similarly, $x \cdot y=x \wedge y$. Hence the elements of $B$ satisfy Ax. 11 and Ax. $11^{\prime}$ with $\vee$ and $\wedge$ replaced by + and - respectively and, as it is known, $B$ thus becomes a Boolean algebra. If $C$ is a subalgebra of $A$ which is also a Boolean algebra with respect to the operations,$+ \cdot$, and - , then every element of $C$ must satisfy the identity $x+x=x$. Hence $C \subseteq B$ and the theorem is proved.

From 1.16 and 1.17 it is seen that with respect to the operations,$+ \cdot$, and - the distinguishing feature between an MV-algebra $\langle A,+, \cdot,-, 0,1\rangle$ and a Boolean algebra is the lack of the idempotent law $x+x=x$, whereas with respect to the operations $\vee, \wedge$, and - the difference between the system $\langle A, \vee, \wedge,-, 0,1\rangle$ and a Boolean algebra is the lack of the law of the excluded middle $x \bigvee \bar{x}=1$. We might mention here that, while various generalizations of the Boolean algebra which do not satisfy the law of the excluded middle are known (e.g., all kind of lattices), there are very few generalizations of the Boolean algebra where the idempotent law does not hold. It is also known that the study of Boolean algebras can be subsumed under the general theory of rings, i.e., the study of the so-called Boolean rings. This transformation is due to certain nice properties of the symmetric difference operator in Boolean algebras. We shall see from the later sections that there will be, in general, no such results for MV-algebras.

To conclude this section we introduce some general procedures to obtain new MV-algebras from those already known $\left({ }^{4}\right)$.

Given an MV-algebra $\langle A,+, \cdot,-, 0,1\rangle$, we say that $B$ is a subalgebra of $A$ if $B \subseteq A, 0,1 \in B, B$ is closed under the operations of,$+ \cdot$, and -. A system $\langle B,+, \cdot,-, 0,1\rangle$ is a homomorphic image of $A$ (or $A$ is homomorphic to $B$ ) if there is a mapping $f$ of $A$ onto $B$ such that $f(0)=0, f(1)=1$, and $f$ preserves the three operations,$+ \cdot$, and -. We say that the function $f$ is a homomorphism of $A$ onto $B$. If the function $f$ is one-to-one, then $f$ if an isomorphism of $A$ onto $B$. In this case we say that the systems $\langle A,+, \cdot,-, 0,1\rangle$ and $\langle B,+, \cdot,-, 0,1\rangle$ are isomorphic. Given a collection of MV-algebras $A_{i}$, $i \in I$, we denote by $P_{i \in I} A_{i}$ the cartesian (or direct) product of the sets $A_{i}$. We denote by $\left\langle P_{i \in I} A_{i},+, \cdot,-, 0,1\right\rangle$ the cartesian (or direct) product of the algebras $A_{i}, i \in I$, where the element 0 is the function $f$ such that $f(i)=0$ for each $i \in I$, the element 1 is the function $f$ such that $f(i)=1$ for each $i \in I$, the addition of two functions $f$ and $g$ shall be the function $h$ such that $h(i)=f(i)$ $+g(i)$ for each $i \in I$, and the product and converse of functions are defined analogously. Due to the form of the axioms Ax. 1-Ax. 11, we see at once that the following is true.

(4) The notions we are about to introduce can be found in their most general form in [7]. 
Theorem 1.18. A subalgebra of an MV-algebra is an MV-algebra, a homomorphic image of an $M V$-algebra is an $M V$-algebra, and the direct product of $M V$-algebras is an $M V$-algebra.

2. Examples of MV-algebras. The first and most important example of an MV-algebra is the algebra $L$ obtained by considering the $\boldsymbol{\aleph}_{0}$-valued propositional calculus. We use here extensively the results and notation in the first three sections of [5]; the formulas and theorems of [5] are referred to by their numbers in parentheses. Let us recall that the formulas in this particular logic are built up of denumerably many statement variables $X_{1}, X_{2}, \cdots, X_{n}, \cdots$ with the two operations $C$ and $N$ in the following manner:

(i) Every statement variable is a formula.

(ii) If $P$ is a formula then $N P$ is a formula.

(iii) If $P$ and $Q$ are formulas then $C P Q$ is a formula.

The particular four axiom schemas are listed below $\left(^{5}\right)$ :

A.1. $C P C Q P$.

A.2. $C C P Q C C Q R C P R$.

A.3. $C C C P Q Q C C Q P P$.

A.4. CCNPNQCQP.

The symbol $\vdash P$ is introduced to mean that the formula $P$ is provable from A.1-A.4 using only modus ponens, i.e., if $P$ and $C P Q$ then $Q$. The elements of our MV-algebra $L$ shall be equivalence classes of formulas determined by the equivalence relation $\equiv$; we let $P / \equiv$ denote the equivalence class one of whose representatives is the formula $P$. It is clear that $[P / \equiv]=[Q / \equiv]$ if and only if $\vdash P \equiv Q$. The operations $+, \cdot,-$ and the elements 0,1 are defined as follows:

$$
\begin{aligned}
& {[P / \equiv]+[Q / \equiv]=[B P Q / \equiv] .} \\
& {[P / \equiv] \cdot[Q / \equiv]=[L P Q / \equiv] .} \\
& {[P / \equiv]^{-}=[N P / \equiv] .} \\
& {[P / \equiv]=1 \text { if and only if } \vdash P .} \\
& {[P / \equiv]=0 \text { if and only if } \vdash N P .}
\end{aligned}
$$

It can be proved without difficulty that these operations on equivalence classes of formulas are independent of the representatives and, hence, are well-defined. In order to see that the system $\langle L,+, \cdot,-, 0,1\rangle$ is an MV-algebra, we note that Ax. 1 and Ax. 2 are given by the commutativity and associativity of $B, A x .3$ by (3.1) and (1.8), Ax. 4 by (3.32), Ax. 5 by (3.45), Ax. 6 by (3.8) and (3.4), and Ax. 7 and Ax. 8 by (3.4). We note also that by (3.4), (1.6), and (1.9), the definitions of the operations $\wedge$ and $\vee$ are such that

(5) The proof that A.5 is derivable from A.1-A.4 can be found in [2]. This fact was also noticed independently by C. A. Meredith a few years ago, and was published in [4]. 


$$
[P / \equiv] \vee[Q / \equiv]=[A P Q / \equiv]
$$

and

$$
[P / \equiv] \wedge[Q / \equiv]=[K P Q / \equiv] \text {. }
$$

Thus Ax. 9, Ax. 10, and Ax. 11 are given by the commutativity of $A$, the associativity of $A$, and the distributive law (3.44) respectively. This takes care of the unprimed axioms; as for the primed axioms, they follow easily from the duality. Hence $L$ is an MV-algebra. In particular, we see that Ax. 1Ax. 11 follow from A. 1-A. 4 upon a proper substitution of the operations $C$ and $N$ by the operations,$+ \cdot$, and - On the other hand, making these same substitutions, we see that A. 1-A. 4 can be transformed equivalently into the following (subject to the condition that $[\bar{x}]^{-}=x$, i.e., Ax. 7 ):

A. $1^{\prime} \cdot \bar{x}+(\bar{y}+x)=1$.

A. $2^{\prime} \cdot(\bar{x}+y)^{-}+\left[(\bar{y}+z)^{-}+(\bar{x}+z)\right]=1$.

A. $3^{\prime} .(x \vee y)^{-}+(y \bigvee x)=1$.

A. $4^{\prime} .(x+\bar{y})^{-}+(\bar{y}+x)=1$.

Clearly A. $1^{\prime}$ and A. $3^{\prime}$ are derivable from Ax. 1-Ax. 11. A. $2^{\prime}$ is simply, after a transformation using the axioms and $1.1,(\bar{x} \bigvee \bar{y})+(y \bigvee z)=1$, which is derivable from 1.6 and 1.8. A. $4^{\prime}$ can be transformed into $(x \vee y)+\bar{y}=1$ which is again derivable from 1.6 and 1.8. Hence we conclude that the two sets of axioms A. 1-A. 4 and Ax. 1-Ax. 11 are equivalent under an appropriate relationship between $C$ and $N$ and + , ', and -.

Another class of examples of MV-algebras is obtained by considering any set $S$ of real numbers between 0 and 1 where $S$ satisfies the following:

(i) $0 \in S$ and $1 \in S$.

(ii) If $x, y \in S$ then $\min (1, x+y) \in S$.

(iii) If $x, y \in S$ then $\max (0, x+y-1) \in S$.

(iv) If $x \in S$ then $1-x \in S$.

If we now define for the elements $x, y \in S, x+y=\min (1, x+y), x \cdot y$ $=\max (0, x+y-1)$, and $\bar{x}=1-x$, then there will be no difficulty in checking that the system $\langle S,+, \cdot,-, 0,1\rangle$ is an MV-algebra. We point out here that the operations $\vee$ and $\wedge$ on $S$ are simply

$$
x \vee y=\max (x, y) \text { and } x \wedge y=\min (x, y),
$$

and the relation $\leqq$ is simply the natural ordering of real numbers.

Various special sets $S$ may be taken which satisfy conditions (i)-(iv), e.g., $S=\{0,1\}, S=$ the interval $[0,1], S=$ the set of all rational numbers between 0 and 1 , and $S=$ the set of all rationals of the form $n / m$ for some fixed positive integer $m$ and $0 \leqq n \leqq m$. For each positive integer $m$ we let $S(m)$ denote this last set of numbers and we notice that the operations + , ', and - in the system $\langle S(m),+, \cdot,-, 0,1\rangle$ are respectively 


$$
\begin{aligned}
& {[p / m]+[q / m]=\min (1,[(p+q) / m]),} \\
& {[p / m] \cdot[q / m]=\max (0,[(p+q-m) / m]),}
\end{aligned}
$$

and

$$
[p / m]^{-}=[(m-p) / m] .
$$

Finally, a somewhat special model of MV-algebras is obtained by considering the following set $C$ of formal symbols:

$$
\begin{aligned}
& 0, c, c+c, c+c+c, \cdots, \\
& 1,1-c, 1-c-c, 1-c-c-c, \cdots .
\end{aligned}
$$

For abbreviation, we define $0 \cdot c=0$ and $n \cdot c=c+c+\cdots+c n$-times and $1-0 \cdot c=1$ and $1-n \cdot c=1-c-c-\cdots-c n$-times. In terms of this convention the rules of addition, multiplication, and complementation are written down as follows:

(i) If $x=n \cdot c$ and $y=m \cdot c$ then $x+y=(m+n) \cdot c$; if $x=n \cdot c$ and $y=1-m \cdot c$, then $x+y=1$ if $m \leqq n$ and $x+y=1-(m-n) \cdot c$ if $n<m$; if $x=1-m \cdot c$ and $y=n \cdot c$, then $x+y=1$ if $m \leqq n$ and $x+y=1-(m-n) \cdot c$ if $n<m$; if $x=1-n \cdot c$ and $y=1-m \cdot c$, then $x+y=1$.

(ii) If $x=n \cdot c$ then $\bar{x}=1-n \cdot c$; if $x=1-n \cdot c$ then $\bar{x}=n \cdot c$.

(iii) $x \cdot y=[\bar{x}+\bar{y}]^{-}$.

One can check easily that the system $\langle C,+, \cdot,-, 0,1\rangle$ thus defined is an MV-algebra by showing that each of the axioms Ax. 1-Ax. 11 is satisfied. We can see this fact more simply if we realize that any finite subset of $C$ can be embedded isomorphically into some MV-algebra $S(m)$ for a sufficiently large $m$. The inclusion relation $\leqq$ in the algebra $C$ can be described as follows: $x \leqq y$ if, and only if, one of the conditions below is satisfied: (i) $x=n \cdot c$ and $y=1-m \cdot c$. (ii) $x=n \cdot c$ and $y=m \cdot c$ where $n \leqq m$. (iii) $x=1-n \cdot c$ and $y=1-m \cdot c$ where $m \leqq n$. It will be seen in the following sections that this MV-algebra $C$ will provide a crucial counter example.

As has already been mentioned at the end of $\$ 1$, any subalgebra, homomorphic image, or direct product of the above given examples will again be an MV-algebra.

\section{Some deeper arithmetical consequences of the axioms.}

In this section we continue the investigation started in $\$ 1$. It turned out that the identity $x \cdot \bar{y}+y=y \cdot \bar{x}+x$, i.e., Ax. 9 , will be used quite frequently in the following theorems and we shall not cite Ax. 9 every time it is used.

TheOREM 3.1. $y \cdot(x+z) \leqq x+y \cdot z$.

Proof. $[y \cdot(x+z)]^{-}+x+y \cdot z=\bar{y}+\bar{x} \cdot \bar{z}+x+y \cdot z=(\bar{y}+y \cdot z)$ $+(x+\bar{x} \cdot \bar{z})=(z+\bar{y} \cdot \bar{z})+(\bar{z}+z \cdot x)=(z+\bar{z})+\bar{y} \cdot \bar{z}+z \cdot x=1$.

Hence by 1.13 the theorem follows. 
Theorem 3.2( $\left.{ }^{6}\right)$. If $x \wedge \bar{y}=0$, then $x+y \cdot z=y \cdot(x+z)$.

Proof. Letting $w=\bar{x} \cdot(\bar{y}+\bar{z})+y \cdot(x+z)$, we see that by 3.1 it is sufficient to prove that $w=1$. Now,

$$
\begin{aligned}
x+w & =(x+\bar{x} \cdot(\bar{y}+\bar{z}))+y \cdot(x+z) \\
& =\bar{y}+\bar{z}+x \cdot y \cdot z+y \cdot(x+z) \\
& =\bar{z}+x \cdot y \cdot z+(\bar{y}+y \cdot(x+z)) \\
& =\bar{z}+x \cdot y \cdot z+x+z+\bar{y} \cdot \bar{x} \cdot \bar{z} \\
& =1
\end{aligned}
$$

and

$$
\begin{aligned}
\bar{y}+w & =(\bar{y}+y \cdot(x+z))+\bar{x} \cdot(\bar{y}+\bar{z}) \\
& =x+z+\bar{y} \cdot \bar{x} \cdot \bar{z}+\bar{x} \cdot(\bar{y}+\bar{z}) \\
& =z+\bar{y} \cdot \bar{x} \cdot \bar{z}+(x+\bar{x} \cdot(\bar{y}+\bar{z})) \\
& =z+\bar{y} \cdot \bar{x} \cdot \bar{z}+\bar{y}+\bar{z}+x \cdot y \cdot z \\
& =1 .
\end{aligned}
$$

From (1) and (2) we obtain

$$
(x+w) \wedge(\bar{y}+w)=1 .
$$

By Ax. 11, (3), and the hypothesis

$$
1=(x \wedge \bar{y})+w=w
$$

and the proof is complete.

Theorem $3.3\left(^{7}\right) .(x+\bar{y}) \bigvee(y+\bar{x})=1$.

Proof.

$$
\begin{aligned}
(x+\bar{y}) & \vee(y+\bar{x})=(x+\bar{y}) \cdot[(y+\bar{x})]-y+\bar{x} \\
= & {[(x+\bar{y}) \cdot \bar{y} \cdot x+\bar{x}]+y } \\
= & (x+\bar{y}) \cdot \bar{y}+\bar{x} \cdot[(x+\bar{y}) \cdot \bar{y}]-+y \\
= & ((x+\bar{y}) \cdot \bar{y}+y)+\bar{x} \cdot(\bar{x} \cdot y+y) \\
= & x+\bar{y}+y \cdot(x+\bar{y})^{-}+\bar{x} \cdot(\bar{x} \cdot y+y) \\
= & \bar{y}+y \cdot \bar{x} \cdot y+(x+\bar{x} \cdot(\bar{x} \cdot y+y)) \\
= & \bar{y}+y \cdot \bar{x} \cdot y+\bar{x} \cdot y+y+x \cdot(\bar{x} \cdot y+y)^{-} \\
= & 1 .
\end{aligned}
$$

(6) This is simply Theorem 3.2 of [5].

(7) This is an algebraic version of the proof of A.5 in [2]. We point out here that most of the results of [5] can be given algebraic proofs as in Theorems 3.2 and 3.3. Similarly, most of our results here can be translated into the notation and language of [5]. 
THEOREM 3.4. If $x \bigvee y=1$, then $x \cdot x \bigvee y \cdot y=1$.

\section{Proof.}

$$
x \cdot x \bigvee y \cdot y=x \cdot x+(\bar{x}+\bar{x}) \cdot y \cdot y .
$$

By using the hypothesis $\bar{x} \wedge \bar{y}=0$ and 3.2 twice,

$$
(\bar{x}+\bar{x}) \cdot y \cdot y=(\bar{x}+\bar{x} \cdot y) \cdot y=\bar{x}+\bar{x} \cdot y \cdot y .
$$

(1) and (2) yield

$$
\begin{aligned}
x \cdot x \vee y \cdot y & =(x \cdot x+\bar{x})+\bar{x} \cdot y \cdot y=(\bar{x} \cdot \bar{x}+x)+\bar{x} \cdot y \cdot y \\
& =\bar{x} \cdot \bar{x}+(x+\bar{x} \cdot y \cdot y)=\bar{x} \cdot \bar{x}+y \cdot y+x \cdot(\bar{y}+\bar{y}) .
\end{aligned}
$$

Again by the hypothesis and 3.2, $x \cdot(\bar{y}+\bar{y})=x \cdot \bar{y}+\bar{y}$. Hence (3) leads to

$$
\begin{aligned}
x \cdot x \vee y \cdot y & =\bar{x} \cdot \bar{x}+y \cdot y+x \cdot \bar{y}+\bar{y} \\
& =\bar{x} \cdot \bar{x}+x \cdot \bar{y}+(y \cdot y+\bar{y}) \\
& =\bar{x} \cdot \bar{x}+x \cdot \bar{y}+\bar{y} \cdot \bar{y}+y \\
& =\bar{x} \cdot \bar{x}+\bar{y} \cdot \bar{y}+(x \cdot \bar{y}+y) \\
& =\bar{x} \cdot \bar{x}+\bar{y} \cdot \bar{y}+(x \vee y) \\
& =1,
\end{aligned}
$$

which is the desired conclusion.

We introduce the following definitions:

Definition 3.5. (i) $0 \cdot x=0$ and $(n+1) \cdot x=n \cdot x+x$.

(ii) $x^{0}=1$ and $x^{n+1}=\left(x^{n}\right) \cdot x$.

Definition 3.6. The order of an element $x$, in symbols ord $(x)$, is the least integer $m$ such that $m \cdot x=1$. If no such integer $m$ exists then $\operatorname{ord}(x)=\infty$.

We obtain easily from 3.5 by induction that

$$
\begin{aligned}
{[n \cdot x]-} & =\bar{x}^{n},\left[x^{n}\right]-=n \cdot \bar{x}, m \cdot(n \cdot x)=(m \cdot n) \cdot x, \\
x^{(m+n)} & =\left(x^{m}\right) \cdot\left(x^{n}\right), \text { and } x^{(m \cdot n)}=\left(x^{m}\right)^{n} .
\end{aligned}
$$

THEOREM 3.7. If $x \bigvee y=1$, then $x^{n} \bigvee y^{n}=1$ for each $n$.

Proof. By an easy induction from 3.4, we see that the hypothesis leads to $x^{\left(2^{m}\right)} \bigvee y^{\left(2^{m}\right)}=1$ for each $m$. Since for each $n$ there exists an $m$ such that $n \leqq 2^{m}$, we see that by $1.9, x^{2^{m}} \leqq x^{n}$ and $y^{2^{m}} \leqq y^{n}$. Using this and 1.8 we obtain immediately $x^{n} \vee y^{n}=1$.

THEOREM 3.8. If $\operatorname{ord}(x \cdot y)<\infty$, then $x+y=1$.

Proof. By the hypothesis, for some $n \geqq 1, n \cdot(x \cdot y)=1$. Hence $(\bar{x}+\bar{y})^{n}=0$. By 3.3

$$
(x+y) \vee(\bar{x}+\bar{y})=1
$$

and by 3.7 


$$
(x+y)^{n} \bigvee(\bar{x}+\bar{y})^{n}=1 .
$$

Thus $(x+y)^{n}=1$. By an easy induction using 1.2 (vi) we see that this last leads to $x+y=1$.

Theorem 3.9. If $\operatorname{ord}(x)>2$, then $\operatorname{ord}(x \cdot x)=\infty$.

Proof. By 3.8.

Definition 3.10. An MV-algebra $A$ is locally finite if, and only if, every element of $A$ different from 0 has a finite order.

Definition 3.11. An MV-algebra $A$ is linearly ordered if, and only if, for every $x, y \in A$, either $x \leqq y$ or $y \leqq x$.

TheOREM 3.12. Every locally finite $M V$-algebra is linearly ordered.

Proof. Let $A$ be locally finite and let $x, y \in A$. We wish to show that either $x+\bar{y}=1$ or $\bar{x}+y=1$. If $x+\bar{y} \neq 1$, then $\bar{x} \cdot y \neq 0$. Hence, by the assumption on $A$, ord $(\bar{x} \cdot y)<\infty$. Now it follows from 3.8 that $\bar{x}+y=1$.

The example $C$ given in $\S 2$ furnishes a counterexample to the converse of 3.12. Clearly $C$ is linearly ordered but ord $(c)=\infty$. It is also easily seen that the only Boolean algebra which is linearly ordered is the two-element Boolean algebra consisting of 0 and 1 alone.

Theorem 3.13. If $A$ is linearly ordered, then $x+z=y+z$ and $x+z \neq 1$ implies $x=y$.

Proof. Since $x+z \neq 1$ and $y+z \neq 1$, we see that $\bar{z} x$ and $\bar{z} y$. Since $A$ is linearly ordered, this means $x \leqq \bar{z}$ and $y \leqq \bar{z}$. We obtain the conclusion immediately from 1.14 .

In the remaining part of this section we shall study intensively some properties of linearly ordered MV-algebras. We shall first introduce the function $d(x, y)$ which plays the role of a distance function. The function $d$ is defined as follows:

$$
d(x, y)=\bar{x} \cdot y+\bar{y} \cdot x .
$$

The reader will recognize that in Boolean algebras the element $d(x, y)$ is simply the familiar symmetric difference of $x$ and $y$. We note here that in Boolean algebras there is another way of defining the symmetric difference of $x$ and $y$, namely, $d^{\prime}(x, y)=(x+y) \cdot(\bar{x}+\bar{y})$. However, the operation $d^{\prime}$ thus defined for MV-algebras would not have the desired property that $d^{\prime}(x, x)=0$. For in the algebra $S(2)$ we easily see that $d^{\prime}(1 / 2,1 / 2) \neq 0$.

Theorem 3.14. (i) $d(x, x)=0, d(x, y)=d(y, x), d(x, y)=d(\bar{x}, \bar{y}), d(x, 0)$ $=x$, and $d(x, 1)=\bar{x}$.

(ii) If $d(x, y)=0$, then $x=y$.

(iii) $d(x, z) \leqq d(x, y)+d(y, z)$.

(iv) $d(x+u, y+v) \leqq d(x, y)+d(u, v)$.

(v) $d(x \cdot u, y \cdot v) \leqq d(x, y)+d(u, v)$. 
Proof. (i) is obvious from the definition. (ii) follows from 1.2 (v). In order to prove (iii), we have to show that

$$
x \cdot \bar{z}+\bar{x} \cdot z \leqq \bar{x} \cdot y+x \cdot \bar{y}+\bar{y} \cdot z+\bar{z} \cdot y .
$$

Now

$$
\begin{aligned}
& {[z \cdot \bar{x}]-+\bar{x} \cdot y+\bar{y} \cdot z=\bar{z}+x+\bar{x} \cdot y+\bar{y} \cdot z} \\
& \quad=(x+\bar{x} \cdot y)+(\bar{z}+\bar{y} \cdot z)=y+x \cdot \bar{y}+\bar{y}+y \cdot \bar{z}=1
\end{aligned}
$$

hence

$$
z \cdot \bar{x} \leqq \bar{x} \cdot y+\bar{y} \cdot z .
$$

In an entirely similar fashion,

$$
x \cdot \bar{z} \leqq x \cdot \bar{y}+y \cdot \bar{z} .
$$

(2) and (3) yield (1) and (iii). Condition (iv) is essentially

(4) $(x+u)^{-} \cdot(y+v)+(x+u) \cdot(y+v)^{-} \leqq \bar{x} \cdot y+\bar{y} \cdot x+\bar{u} \cdot v+\bar{v} \cdot u$

Now

$$
\begin{aligned}
& (x+u+\bar{y} \cdot \bar{v})+\bar{x} \cdot y+\bar{u} \cdot v=(x+\bar{x} \cdot y)+\bar{y} \cdot \bar{v}+(u+\bar{u} \cdot v) \\
& \quad=(y+x \cdot \bar{y})+\bar{y} \cdot \bar{v}+(v+u \cdot \bar{v})=x \cdot \bar{y}+u \cdot \bar{v}+v+(y+\bar{y} \cdot \bar{v}) \\
& \quad=x \cdot \bar{y}+u \cdot \bar{v}+v+\bar{v}+y \cdot v=1
\end{aligned}
$$

which implies

$$
(x+u)^{-} \cdot(y+v) \leqq \bar{x} \cdot y+\bar{u} \cdot v .
$$

Similarly, we derive

$$
(x+u) \cdot(y+v)^{-} \leqq \bar{y} \cdot x+\bar{v} \cdot u .
$$

(5) and (6) give (4) and (iv). As for condition (v), we note that

$$
\begin{aligned}
d(x \cdot u, y \cdot v) & =d\left([\bar{x}+\bar{u}]^{-},[\bar{y}+\bar{v}]^{-}\right)=d(\bar{x}+\bar{u}, \bar{y}+\bar{v}) \\
& \leqq d(\bar{x}, \bar{y})+d(\bar{u}, \bar{v})=d(x, y)+d(u, v) .
\end{aligned}
$$

The theorem is proved.

3.14 tells us that many of the properties of the symmetric difference operation in Boolean algebras can be carried over to MV-algebras. However, two of the most important properties of symmetric difference, i.e., associativity and the distribution of multiplication over symmetric difference, fail. This can be seen, for instance, in the MV-algebra $S(3)$, where $d(1 / 3, d(2 / 3,1))$ $\neq d(d(1 / 3,2 / 3), 1)$, and in the MV-algebra $S(2)$, where $(1 / 2) \cdot d(1 / 2,1)$ $\neq d(1 / 2 \cdot 1 / 2,1 / 2 \cdot 1)$. This is the main reason why we pointed out in $\$ 1$ that the study of $\mathrm{MV}$-algebras cannot be subsumed under the theory of rings with the operation $d$ interpreted as the ring addition. However, this does not preclude the possibility that some other definition of ring addition may work. 
In this connection, we easily see that the operation $d^{\prime}$ introduced earlier is also such that $d^{\prime}\left(1 / 3, d^{\prime}(2 / 3,1)\right) \neq d^{\prime}\left(d^{\prime}(1 / 3,2 / 3), 1\right)$ in $S(3)$ and $(1 / 2)$ $\cdot d^{\prime}(1 / 2,1) \neq d^{\prime}(1 / 2 \cdot 1 / 2,1 / 2 \cdot 1)$ in $S(2)$.

Theorem 3.15. If $x \leqq y$, then $d(x, y)=\bar{x} \cdot y$ and $x+d(x, y)=y$.

Proof. If $x \leqq y$ then $x \cdot \bar{y}=0$. Thus $d(x, y)=\bar{x} \cdot y$. Also, if $x \leqq y$, then $y=x \bigvee y$ $=x+\bar{x} \cdot y=x+d(x, y)$.

THEOREM 3.16. (i) If $n \cdot y \leqq x \leqq(n+1) \cdot y$, then $d(x, n \cdot y) \leqq y$ and $d(x,(n+1) \cdot y) \leqq y$.

(ii) If $n \cdot y \leqq x<(n+1) \cdot y$, then $d(x, n \cdot y)<y$.

(iii) If $n \cdot y<x \leqq(n+1) \cdot y$, then $d(x,(n+1) \cdot y)<y$.

Proof. If $n \cdot y \leqq x \leqq(n+1) \cdot y$, then $(n \cdot y) \cdot \bar{x}=0$ and $x \cdot\left(\bar{y}^{(n+1)}\right)=0$. Also $d(x, n \cdot y)=x \cdot \bar{y}^{n}$ and $d(x,(n+1) \cdot y)=\bar{x} \cdot((n+1) \cdot y)$. From $x \cdot\left(\bar{y}^{(n+1)}\right)=0$, we obtain $\left(x \cdot \bar{y}^{n}\right) \cdot \bar{y}=0$, thus $d(x, n \cdot y) \leqq y$. From $\bar{x} \cdot(n \cdot y)=0$ and 3.1 , we see that $d(x,(n+1) \cdot y)=\bar{x} \cdot(y+n \cdot y) \leqq y+\bar{x} \cdot(n \cdot y)=y$. Hence (i) is proved. If $d(x, n \cdot y)=y$, then $x \cdot \bar{y}^{n}=y$ and $x=x+0=x+(n \cdot y) \cdot \bar{x}=n \cdot y+\bar{y}^{n} \cdot x=n \cdot y+y$ $=(n+1) \cdot y$. This proves (ii). If $d(x,(n+1) \cdot y)=y$, then $\bar{x} \cdot((n+1) \cdot y)=y$ and $\bar{x}=\bar{x}+0=\bar{x}+x \cdot \bar{y}^{(n+1)}=\bar{y}^{(n+1)}+x \cdot((n+1) \cdot y)=\bar{y}^{n+1}+y=\bar{y}^{n} \cdot \bar{y}+y$ $=\bar{y}^{n}+y \cdot(n \cdot y) \geqq \bar{y}^{n}$. By $1.4(\mathrm{vi})$, this means $x \leqq n \cdot y$. This proves (iii). The proof is complete.

TheOREM 3.17. If $A$ is linearly ordered and $\operatorname{ord}(y)=m<\infty$, then $d([n \cdot y]-,(m-n) \cdot y)<y$ for each $n \leqq m$.

Proof. The theorem is clearly true if $n=m$. Therefore let us assume $n<m$. In this case $(m-n) \cdot y+n \cdot y=1$, hence $[n \cdot y]-\leqq(m-n) \cdot y$. On the other hand, if $[n \cdot y]-\leqq(m-n-1) \cdot y$, then $1=(m-n-1) \cdot y+n \cdot y=(m-1) \cdot y$ which contradicts ord $(y)=m$. Thus we have $(m-n-1) \cdot y<[n \cdot y]-\leqq(m-n)$ $\cdot y$. The conclusion now follows from 3.16.

Definition 3.18. An element $x$ of an MV-algebra $A$ is an atom if, and only if, $x \neq 0$ and whenever $0 \leqq y \leqq x$, then either $y=0$ or $y=x$.

The following theorem describes completely those MV-algebras which are locally finite and which contain an atom.

ThEOREM 3.19. If $A$ is linearly ordered and contains an atom of order $m$, then $A$ is isomorphic to $S(m)$.

Proof. Let $y$ be an atom of $A$ of order $m$ and we see by 3.13 that

$$
0<y<2 \cdot y<\cdots<(m-1) \cdot y<m \cdot y=1 \text {. }
$$

Since $A$ is linearly ordered it follows that any element $x \neq 1$ is such that $n \cdot y \leqq x<(n+1) \cdot y$ for some $n \leqq m-1$. From 3.16(ii) we have that $d(x, n \cdot y)<y$ and, since $y$ is an atom, $d(x, n \cdot y)=0$. This, by 3.14(ii), implies $x=n \cdot y$. We have now proved that any element must be a multiple of $y$. By $3.17, d\left((n \cdot y)^{-}\right.$, 
$(m-n) \cdot y)<y$ and, again since $y$ is an atom, $[n \cdot y]-=(m-n) \cdot y$. Clearly $n \cdot y+l \cdot y=(n+l) \cdot y$. It is now evident that the function $f$ defined by $f(n \cdot y)$ $=n / m$ will map $A$ isomorphically onto $S(m)$ and the theorem is proved.

There now remains the more difficult situation of $\mathrm{MV}$-algebras containing no atoms.

THEOREM 3.20. If $A$ is linearly ordered and contains no atoms, then for any $x \neq 0$ and for any $n$ there exists $a y \neq 0$ for which $n \cdot y \leqq x$.

Proof. We shall first prove the following

Lemma. If $A$ is linearly ordered and $x \leqq y$, then either $x+x \leqq y$ or $d(x, y)$ $+d(x, y) \leqq y$.

Assume that not $x+x \leqq y$. Since $A$ is linearly ordered, $y \leqq x+x$ and $1=\bar{y}+x+x=[y \cdot \bar{x}]-+x$. Thus $d(x, y)=\bar{x} \cdot y \leqq x$. By 3.15 we see that $d(x, y)$ $+d(x, y) \leqq x+d(x, y)=y$. The lemma is proved.

Now the theorem will be proved by induction on $n$. Clearly it holds for $n=1$. Assume that the theorem holds for $n$ and $x, y$ are such that $y \neq 0$ and $n \cdot y \leqq x$. Since $A$ contains no atoms, there exists a $z$ such that $0<z<y$. By letting $w=z$ or $w=d(z, y)$, we see from our lemma that $w \neq 0$ and $w+w \leqq y$. It is now easy to see that $(n+1) \cdot w \leqq(2 \cdot n) \cdot w \leqq n \cdot y \leqq x$ and $w$ is the desired element.

Theorem 3.21. Let $A$ be locally finite and contain no atoms. Then for any two elements $x, y \in A$ for which $x<y$, there exists an element $z \in A$ such that $x<z<y$.

Proof. Suppose that $x<y$, then, by $3.15, x+d(x, y)=y$ where $d(x, y) \neq 0$. If $d(x, y)=1$, then, again by $3.15, \bar{x} \cdot y=1$ and, by $1.2(\mathrm{vi}), x=0$ and $y=1$. In this case by our hypothesis, clearly there will exist an element $z$ such that $x<z<y$. Thus, let us assume that $0 \neq d(x, y)$ and $1 \neq d(x, y)$. By 3.12 and 3.20 we see that there exists an element $w$ such that $0 \neq w, w \leqq d(x, y)$, and $w+w \leqq d(x, y)$. Since $A$ is locally finite, $w<d(x, y)$. For otherwise $d(x, y)$ $=d(x, y)+d(x, y)$ and $\operatorname{ord}(d(x, y))=\infty$. Thus we have that $0<w<d(x, y)$. Consider now the element $z=x+w$. Clearly $x \leqq z \leqq y$. Now, if $z=y$, then $x+w \leqq x+w+w \leqq x+d(x, y)=y \leqq x+w$. Hence, $x+w=x+w+w$. If $y=1$, then $\bar{d}(x, y)=\bar{x}, w \leqq \bar{x}, w+w \leqq \bar{x}$, and, by $1.14, w=w+w$. This, of course is a contradiction to the fact that ord $(w)<\infty$. If $y \neq 1$, then, by 3.12 and 3.13 , again we arrive at the contradiction $w=w+w$. Thus we see that $z<y$. Now, if $x=z$, then $x=x+w$. Since we already know that $z<y$ and $z \neq 1$, by 3.13 , we obtain the contradiction $w=0$. Thus $x<z$. The theorem has been proved.

From 3.21 we see that if $A$ is locally finite and contains no atoms, then $A$ is densely ordered. Now a theorem which would correspond to 3.19 for this case might go somewhat like this: If $A$ is denumerable, locally finite, and contains no atoms, then $A$ is isomorphic to a subalgebra of the MV- 
algebra of all real numbers between 0 and 1 . So far we have been unable to prove or disprove this conjecture. It seems to be a difficult question. In this connection the following example suggested by $\mathrm{J}$. B. Rosser is of some interest. It is essentially an MV-algebra which is a generalization of the algebra $C$ discussed in $\$ 2$ and which is densely ordered but not locally finite. Let $D$ be the set of all formal symbols of the form

$\begin{array}{cc}n \cdot c_{0}, & 1-n \cdot c_{0}, \\ n \cdot c_{1}, & 1-n \cdot c_{1}, \\ \cdots & \cdots \\ n \cdot c_{p}, & 1-n \cdot c_{p}, \\ \cdots & \cdots,\end{array}$

with the stipulation that for $0 \leqq p \leqq q$, we have the relation $c_{p}=2^{(q-p)} \cdot c_{q}$. Now, given any two elements $x$ and $y$ of $D$, we first reduce these elements by the above given relations to forms where they both involve a common $c_{p}$ and then we imitate the definition of,$+ \cdot$, and - as for the case of the algebra $C$. It is again easily checked that $D$ under these definitions of,$+ \cdot$, and - is an MV-algebra. Furthermore, $D$ is obviously densely ordered. However, the element $c_{0}$ has infinite order. This example, of course, shows that our conjecture stated above would be false if the phrase "locally finite" is replaced by "densely ordered."

The following is simply an attempt to throw the whole situation of MValgebras which are locally finite and atomless back into the finite cases $S(m)$.

For the subsequent discussion, we introduce the notion of a polynomial function of $s$ variables $v_{1}, v_{2}, \cdots, v_{s}$. Since, by the duality, the operation is definable in terms of + and -, we shall restrict our discussion to polynomials built up from + and - only. We point out here that in the following definition two polynomial functions are equal if and only if they are identical.

Definition 3.22. (i) $P\left(v_{1}, \cdots, v_{s}\right)$ is a polynomial function of rank 1 if $P\left(v_{1}, \cdots, v_{s}\right)=v_{j}$ for some $j, 1 \leqq j \leqq s$.

(ii) $P\left(v_{1}, \cdots, v_{8}\right)$ is a polynomial function of rank $(m+1)$ if either $P=\bar{Q}$ where $Q$ is a polynomial function of rank $m$, or else $P=Q+R$ where $Q$ and $R$ are polynomial functions of rank $\leqq m$ and at least one of the polynomials $Q$ or $R$ is of rank $m$.

(iii) $P$ is a polynomial function if, and only if, for some $m P$ is a polynomial function of rank $m$.

It is quite clear that if $x_{1}, \cdots, x_{8}$ are elements of $A$, then $P\left(x_{1}, \cdots, x_{8}\right)$ is also an element of $A$.

Theorem 3.23. Let $x_{1}, \cdots, x_{8}$ be elements of $A$, let $n_{1}, \cdots, n_{s}$ be an arbitrary sequence of integers, and let $y_{i}=n_{i} \cdot y$ for some fixed $y$ and $1 \leqq i \leqq s$. Suppose further that $d\left(x_{i}, y_{i}\right) \leqq y$ for $1 \leqq i \leqq s$, then, for whatever polynomial function $P$ of $s$ variables and rank $\leqq(m+1), d\left(P\left(x_{1}, \cdots, x_{s}\right), P\left(y_{1}, \cdots, y_{s}\right)\right) \leqq 2^{m} \cdot y$. 
Proof. By induction on $m$. The theorem is clearly true for $m=0$. Assume now that the theorem holds for $m$. Let $P$ be a polynomial function of rank $\leqq m+2$ and we have two cases:

CASE 1. $P=\bar{Q}$ where $Q$ is of rank $\leqq m+1$. By the inductive hypothesis $d\left(Q\left(x_{1}, \cdots, x_{s}\right), Q\left(y_{1}, \cdots, y_{s}\right)\right) \leqq 2^{m} \cdot y . \quad$ By 3.14(i), $d\left(P\left(x_{1}, \cdots, x_{s}\right)\right.$, $\left.P\left(y_{1}, \cdots, y_{s}\right)\right)=d\left(\bar{Q}\left(x_{1}, \cdots, x_{s}\right), \quad \bar{Q}\left(y_{1}, \cdots, y_{s}\right)\right)=d\left(Q\left(x_{1}, \cdots, x_{s}\right)\right.$, $\left.Q\left(y_{1}, \cdots, y_{s}\right)\right) \leqq 2^{m} \cdot y \leqq 2^{(m+1)} \cdot y$.

CASE 2. $P=Q+R$ where $Q$ and $R$ are of ranks $\leqq m+1$. By the inductive hypothesis and 3.14 (iv),

$$
\begin{gathered}
d\left(P\left(x_{1}, \cdots, x_{s}\right), P\left(y_{1}, \cdots, y_{s}\right)\right)=d\left(Q\left(x_{1}, \cdots, x_{s}\right)+R\left(x_{1}, \cdots, x_{s}\right),\right. \\
\left.Q\left(y_{1}, \cdots, y_{s}\right)+R\left(y_{1}, \cdots, y_{s}\right)\right) \leqq d\left(Q\left(x_{1}, \cdots, x_{s}\right), Q\left(y_{1}, \cdots, y_{s}\right)\right) \\
\quad+d\left(R\left(x_{1}, \cdots, x_{s}\right), R\left(y_{1}, \cdots, y_{s}\right)\right) \leqq 2^{m} \cdot y+2^{m} \cdot y=2^{(m+1)} \cdot y .
\end{gathered}
$$

The induction is complete and the theorem is proved.

From $3.12,3.16,3.20$, and 3.23 we see readily that:

Theorem 3.24. Let $A$ be locally finite and contain no atoms. Then, for every polynomial function $P$ of $s$ variables, for any elements $x_{1}, \cdots, x_{s} \in A$, and for any element $x$ of $A$ different from 0 , there exists an element $y$ of $A$ and a sequence of integers $n_{1}, \cdots, n_{s}$ such that

$$
d\left(P\left(x_{1}, \cdots, x_{8}\right), P\left(n_{1} \cdot y, \cdots, n_{s} \cdot y\right)\right) \leqq x .
$$

Consider an element $y$ of finite order $m$ and the set of multiples of $y$, $n \cdot y$, for $0 \leqq n \leqq m$. We define the operation ${ }^{-1}$ for this set of elements as follows:

$$
(n \cdot y)^{-\prime}=(m-n) \cdot y .
$$

Now, for any polynomial function $P$ we let $P^{\prime}$ be the resulting polynomial function built up of + and $^{-1}$ where we replace each - in $P$ by ${ }^{-1}$. If $n_{1}, \cdots, n_{s}$ is a sequence of integers, then clearly $P^{\prime}\left(n_{1} \cdot y, \cdots, n_{s} \cdot y\right)$ will again be an element of the form $n \cdot y$ for some $n$. Also, it follows readily that $(Q+R)^{\prime}$ $=Q^{\prime}+R^{\prime}$ and $(\bar{Q})^{\prime}=\left[\bar{Q}^{\prime}\right]^{-\prime}$. Notice that we are essentially trying to pretend that the element $y$ in some way behaves like the generator $1 / m$ in the algebra $S(m)$. We have the following approximation theorem:

Theorem 3.25. If $A$ is linearly ordered and ord $(y)=m$, then for any polynomial $P$ of $s$ variables and rank $\leqq(n+1)$ and for any sequence of integers $n_{1}, \cdots, n_{s}$,

$$
d\left(P\left(n_{1} \cdot y, \cdots, n_{s} \cdot y\right), P^{\prime}\left(n_{1} \cdot y, \cdots, n_{s} \cdot y\right)\right) \leqq 2^{n} \cdot y .
$$

Proof. By induction on $n$. We shall let $y_{i}=n_{i} \cdot y$ for $1 \leqq i \leqq s$. Clearly the theorem holds for $n=0$. Assume that the theorem holds for $n$ and let $P$ be a polynomial function of rank $\leqq n+2$. 
CASE 1. $P=\bar{Q}$ where $Q$ has rank $\leqq n+1$. In this case by 3.14(i) and (iii)

$$
\begin{aligned}
d\left(P \left(y_{1}, \cdots,\right.\right. & \left.\left.y_{s}\right), P^{\prime}\left(y_{1}, \cdots, y_{s}\right)\right) \\
& =d\left(\bar{Q}\left(y_{1}, \cdots, y_{s}\right),\left[Q^{\prime}\right]^{-\prime}\left(y_{1}, \cdots, y_{s}\right)\right) \\
& \leqq d\left(\bar{Q}\left(y_{1}, \cdots, y_{8}\right),\left[Q^{\prime}\right]^{-}\left(y_{1}, \cdots, y_{s}\right)\right) \\
& +d\left(\left[Q^{\prime}\right]-\left(y_{1}, \cdots, y_{s}\right),\left[Q^{\prime}\right]^{-\prime}\left(y_{1}, \cdots, y_{s}\right)\right) .
\end{aligned}
$$

By 3.14(i) and the inductive hypothesis,

$$
\begin{aligned}
d\left(\bar{Q}\left(y_{1}, \cdots, y_{s}\right),\left[Q^{\prime}\right]-\left(y_{1}, \cdots, y_{s}\right)\right) & \\
& =d\left(Q\left(y_{1}, \cdots, y_{s}\right), Q^{\prime}\left(y_{1}, \cdots y_{s}\right)\right) \leqq 2^{n} \cdot y .
\end{aligned}
$$

On the other hand, by 3.17 ,

$$
d\left(\left[Q^{\prime}\right]-\left(y_{1}, \cdots, y_{s}\right),\left[Q^{\prime}\right]^{-\prime}\left(y_{1}, \cdots, y_{s}\right)\right) \leqq y .
$$

Hence, it follows from (1), (2), and (3) that

$$
d\left(P\left(y_{1}, \cdots, y_{s}\right), P^{\prime}\left(y_{1}, \cdots, y_{s}\right)\right) \leqq 2^{n} \cdot y+y \leqq 2^{(n+1)} \cdot y .
$$

CASE 2. $P=Q+R$ where $Q$ and $R$ have ranks $\leqq(n+1)$. By a straightforward calculation using 3.14(iv) and the inductive hypothesis,

$$
\begin{aligned}
& d\left(P\left(y_{1}, \cdots, y_{s}\right), P^{\prime}\left(y_{1}, \cdots, y_{s}\right)\right) \\
& \quad=d\left(Q\left(y_{1}, \cdots, y_{s}\right)+R\left(y_{1}, \cdots, y_{s}\right), Q^{\prime}\left(y_{1}, \cdots, y_{s}\right)+R^{\prime}\left(y_{1}, \cdots, y_{s}\right)\right) \\
& \left.\quad \leqq d\left(Q\left(y_{1}, \cdots, y_{s}\right), Q^{\prime}\left(y_{1}, \cdots, y_{s}\right)\right)+d\left(R\left(y_{1}, \cdots, y_{s}\right), R^{\prime}\left(y_{1}, \cdots, y_{s}\right)\right)\right) \\
& \quad \leqq 2^{n} \cdot y+2^{n} \cdot y=2^{(n+1)} \cdot y .
\end{aligned}
$$

Thus the induction is complete and the theorem is proved.

Combining 3.24 and 3.25 and with the use of 3.14(iii), we obtain

Theorem 3.26. Let $A$ be locally finite and contain no atoms. Then, for every polynomial $P$ of $s$ variables, for any elements $x_{1}, \cdots, x_{s}$ of $A$, and for any element $x$ of $A$ different from 0 , there exists an element $y$ of $A$ and a sequence of integers $n_{1}, \cdots, n_{s}$ such that $\left.d\left(P x_{1}, \cdots, x_{s}\right), P^{\prime}\left(n_{1} \cdot y, \cdots, n_{s} \cdot y\right)\right) \leqq x$.

At first sight these approximation theorems seem very crude and inelegant. However, without a proof of the conjecture mentioned after 3.21 we see at present no other way of obtaining our results 5.3 and 5.4 in $\$ 5$.

\section{Ideals, congruence relations, and the problem of representation.}

Definition 4.1. A subset $I$ of $A$ is an ideal of $A$ if, and only if, (i) $0 \in I$, (ii) if $x, y \in I$, then $x+y \in I$, and (iii) if $x \in I$ and $y \leqq x$, then $y \in I$.

An ideal $I$ is said to be proper if $I \neq A$. Clearly an ideal $I$ is proper if, and only if, $1 \notin I$.

Definition 4.2. $R$ is a congruence relation over $A$ if, and only if (i) $R$ is an equivalence relation over $A$ and (ii) if $x R y$ and $u R v$, then $\bar{x} R \bar{y}$, $x+u R y+v$, and $x \cdot u R y \cdot v$.

The definition of a congruence relation $R$ is such that $R$ automatically 
preserves the operations $\vee$ and $\wedge$. As usual we let $x / R$ denote the coset of the equivalence relation $R$ determined by $x$; we let $A / R$ denote the set of all cosets of $R$. It may also be pointed out here that, just as in the case of Boolean algebras, 4.1(iii) may be replaced by 4.1(iii'): If $x \in I$ and $y \in A$, then $x \cdot y \in I$. It is clear that 4.1 (iii) implies $4.1\left(\right.$ iii $\left.^{\prime}\right)$. On the other hand,

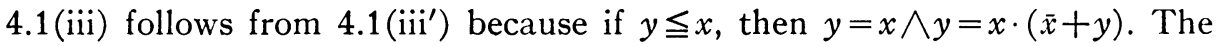
following is a theorem that we would expect concerning the connection between ideals, homomorphisms, and congruence relations.

Theorem 4.3. (i) If $f$ is a homomorphism of $A$ onto another $M V$-algebra, then the set of elements $x \in A$ such that $f(x)=0$ is an ideal and the relation $R$ defined by $x R y$ if and only if $f(x)=f(y)$ is a congruence relation.

(ii) If $R$ is a congruence relation, then the set of elements of $0 / R$ is an ideal and the set of all cosets of $R$ forms an $M V$-algebra $\langle A / R,+, \cdot,-, 0 / R, 1 / R\rangle$ under the definitions $(x / R)+(y / R)=(x+y) / R,(x / R) \cdot(y / R)=(x \cdot y) / R$, and $[(x / R)]^{-}=\bar{x} / R$, where $A / R$ is an homomorphic image of $A$ under the mapping $f(x)=x / R$.

(iii) If $R$ is a congruence relation, then $x R$ if and only if $d(x, y) R 0$.

(iv) If $R$ and $S$ are congruence relations, then $R=S$ if and only if $0 / R$ $=0 / S$.

(v) If $I$ is an ideal, then the relation $R$ defined by $x R y$ if and only if $d(x, y) \in I$ is a congruence relation.

Proof. (i) and (ii) are obvious. (iii) is obtained as follows. Assume that $x R y$, then since $\bar{x} R \bar{x}$ and $\bar{y} R \bar{y}$, we obtain $x \cdot \bar{y} R 0, \bar{x} \cdot y R 0$, and $(x \cdot \bar{y}+y \cdot \bar{x}) R 0$. Hence $d(x, y) R 0$. Assume now $d(x, y) R 0$. Since $(x \cdot \bar{y}+0)$ $R(x \cdot \bar{y}+0)$,

$$
[(x \cdot \bar{y}+0) \wedge(x \cdot \bar{y}+y \cdot \bar{x})] R[(x \cdot \bar{y}+0) \wedge 0]
$$

and, by Ax. 11,

$$
[x \cdot \bar{y}+(0 \wedge(y \cdot \bar{x}))] R 0 .
$$

This implies $x \cdot \bar{y} R 0$. Similarly $y \cdot \bar{x} R 0$. Now, $(y+0) R(y+x \cdot \bar{y}),(x+y \cdot \bar{x}) R$ $(x+0), y+x \cdot \bar{y}=x+y \cdot \bar{x}$, and $x R y$. (iv) follows directly from (iii). As for (v), it is simply a consequence of 3.14 .

4.3 tells us that there is a one-to-one correspondence between the ideals of $A$ and the congruence relations over $A$. Thus in our discussion we can use either notion interchangeably. In particular, given an ideal $I$ we write $x / I$ $=x / R$ and $A / I=A / R$ where $R$ is the unique congruence relation associated with $I$.

The familiar theorem on principal ideals in Boolean algebra reduces to the following:

Theorem 4.4. There exists a proper ideal I such that $x \in I$ if, and only if, $\operatorname{ord}(x)=\infty$. 
Proof. Clearly if $x$ belongs to a proper ideal, then $\operatorname{ord}(x)=\infty$. On the other hand, if $\operatorname{ord}(x)=\infty$, then we take $I$ to be the set of all elements $y$ such that $y \leqq m \cdot x$ for some $m$. It then is obvious that $I$ is a proper ideal.

Thus we see that, in general, not every element $x \neq 1$ can be a member of a proper ideal.

Definition 4.5. (i) $M$ is a maximal ideal of $A$ if, and only if, $M$ is a proper ideal and whenever $I$ is an ideal such that $M \subseteq I \subseteq A$, then either $M=I$ or $I=A$.

(ii) $R$ is a maximal congruence relation of $A$ if, and only if, $R$ is not the trivial congruence relation over $A$ (i.e., $R \neq A^{2}$ ) and whenever $S$ is a congruence relation such that $R \subseteq S \subseteq A^{2}$, then either $R=S$ or $S=A^{2}$.

It can easily be seen that the previously mentioned one-to-one correspondence between ideals and congruence relations is such that maximal ideals correspond to maximal congruence relations and vice-versa.

THEOREM 4.6. Every proper ideal can be extended to a maximal ideal.

Proof. Usual proof using the Axiom of Choice and the fact that the element 1 never belongs to a proper ideal.

Theorem 4.7. Let $M$ be an ideal of $A$, then the following conditions are equivalent:

(i) $M$ is a maximal ideal.

(ii) For every element $x \notin M, \bar{x}^{n} \in M$ for some $n$.

(iii) $A / M$ is locally finite.

Proof. Assume (i) and let $x$ be such that $x \notin M$. Let $I$ be defined as the set of all $t$ 's such that for some $y \in M$ and for some $n, t \leqq y+n \cdot x$. Clearly $I$ is an ideal and $M \subseteq I$. Since $x \in I$ and $x \in M$, we see that $I=A$ and $1 \in I$. This means that for some $y \in M$ and some $n, 1=y+n \cdot x$. This last leads to $[n \cdot x]^{-}$ $\leqq y, \bar{x}^{n} \leqq y$, and $\bar{x}^{n} \in M$. Assume (ii) and let $x / M \in A / M$ be such that $0 / M$ $\neq x / M$, i.e., $x \in M$. Thus, for some $n, \bar{x}^{n} \in M$ and $(0 / M)=\left(\bar{x}^{n} / M\right)=(\bar{x} / M)^{n}$ $=\left([x / M]^{-}\right)^{n}$. This last implies that $n \cdot(x / M)=1 / M$. Assume (iii) and let $I$ be any ideal for which $M \subseteq I$ and there exists an element $x$ such that $x \in I$ and $x \notin M$. Thus, for some $n, n \cdot(x / M)=1 / M$. Since $x \in I, n \cdot x / I=0 / I$, and since $M \subseteq I, n \cdot x / I=1 / I$. This gives $0 / I=1 / I$ and $I=A$.

Due to 4.7 we see that the class of locally-finite $M V$-algebras which we introduced in $\$ 3$ corresponds to precisely the class of so-called simple MValgebras, i.e., those MV-algebras $A$ in which the only ideals are the sets $\{0\}$ and $A$. Also this fact can be seen easily and directly from the definitions. It is known that Boolean algebras can always be represented as algebras of sets, i.e., where the operations,$+ \cdot$, and - can always be interpreted as the union of set, the intersection of sets, and the complementation of sets with respect to a given unit $\left.{ }^{8}\right)$. Here for MV-algebras we are at a loss to see what, if any-

(8) Cf., [6]. 
thing, a natural representation means. However, algebraically the Boolean representation theorem can be equivalently stated in the following:

$\left(^{*}\right)$ Every Boolean algebra is isomorphic to a subalgebra of a direct product of simple Boolean algebras (i.e., the two-element Boolean algebra).

Taking our cue from $\left(^{*}\right)$ we now define representable MV-algebras as follows:

Definition 4.8. An MV-algebra is representable if, and only if, it is isomorphic to a subalgebra of a direct product of locally finite MV-algebras.

It easily follows from our discussion of ideals and congruence relations and from [1, Theorem 9, p. 92], adapted to our special case of MV-algebras that the class of representable $\mathrm{MV}$-algebras can be characterized as follows:

TheOREM 4.9. An MV-algebra $A$ is representable if, and only if, the intersection of all maximal ideals of $A$ is the set $\{0\}$.

The question now remaining is whether every MV-algebra is representable. The answer is no. Take very simply the MV-algebra $C$ discussed in $\$ 2$. It is easily seen that $C$ has only one maximal ideal which is the set of all elements of the form $n \cdot c$ for some $n$; thus it follows from 4.9 that $C$ is not representable in the sense of 4.8 . It should be mentioned here that the characterization given in 4.9 is the same as the statement for every $x \neq 0$, there is a maximal ideal $M$ such that $x \notin M$. This latter condition is of course satisfied in every Boolean algebra.

If we now let $K$ denote the class of representable $\mathrm{MV}$-algebras, then by the example $C$ mentioned above we see that the class $K$ is a proper subclass of the class of all MV-algebras. The natural question to ask here is whether the class $K$ is an equational class, i.e., whether $K$ can be characterized by a set of equations in addition to those already given by the axioms. The answer is again no. Using the terminology of [7], we state and prove the following which is a stronger statement than the fact that $K$ is not equational.

Theorem 4.10. The class $K$ is not a universal class.

Proof. It has already been remarked that the MV-algebra $C$ is not representable. Also, every finite subset of $C$ can be isomorphically embedded in some representable MV-algebra $S(m)$ for some $m$. Thus it follows from the characterization given in [7] that $K$ is not a universal class.

5. Applications to the MV-algebra $L$ and some related results. Let us now return to the algebra $L$ introduced in $\$ 2$. For the sake of uniformity of notation, we now let $x, y, z, \cdots$ denote the elements of $L$. The generators of $L$ shall be denoted by capital letters $X_{1}, \cdots, X_{n}, \cdots$. We understand that any set of capital letters like $\left\{Y_{1}, \cdots, Y_{s}\right\},\left\{Z_{1}, \cdots, Z_{p}\right\}$, or $\left\{W_{1}, \cdots, W_{q}\right\}$ shall denote a subset of the set of generators of $L$.

For the discussion in the succeeding paragraphs, we let the symbol $S(\infty)$ denote the set of rational numbers between 0 and 1 , and the system 
$\langle S(\infty),+, \cdot,-, 0,1\rangle$ shall denote the MV-algebra formed from the set $S(\infty)$. Given the generators $Y_{1}, \cdots, Y_{s}$ of $L$, an assignment $h$ of $Y_{1}, \cdots, Y_{s}$ into $S(\infty)$ is simply a mapping $h$ such that $h\left(Y_{i}\right) \in S(\infty)$ for $1 \leqq i \leqq s$. An element $x=P\left(Y_{1}, \cdots, Y_{s}\right)$ of $L$ is valid in case $P\left(h\left(Y_{1}\right), \cdots, h\left(Y_{s}\right)\right)=1$ for every assignment $h$. In terms of these notions, the completeness of the $\boldsymbol{\aleph}_{0}$-valued logic can then be stated in the following

TheOREM 5.1. Let $\left\{Y_{1}, \cdots, Y_{s}\right\}$ be any finite subset of the generators of $L$. Then for any polynomial function $P\left(v_{1}, \cdots, v_{s}\right), P\left(Y_{1}, \cdots, Y_{s}\right)=1$ if and only if $P$ is valid.

Since $L$ is generated by the generators $X_{1}, \cdots, X_{n}, \cdots$, we see without difficulty that every element $x$ of $L$ can be represented as $x=P\left(Y_{1}, \cdots, Y_{8}\right)$ for some polynomial $P$ and some set of generators $\left\{Y_{1}, \cdots, Y_{s}\right\}$. The next theorem expresses a uniqueness property of this representation.

Theorem 5.2. If an element $x \in L$ can be represented as $x=P\left(Y_{1}, \cdots, Y_{s}\right)$ and $x=Q\left(Y_{1}, \cdots, Y_{s}\right)$, then for every assignment $h, P\left(h\left(Y_{1}\right), \cdots, h\left(Y_{s}\right)\right)$ $=Q\left(h\left(Y_{1}\right), \cdots, h\left(Y_{s}\right)\right)$.

Proof. By the hypothesis, we see that

$$
\left[(\bar{P}+Q)\left(Y_{1}, \cdots, Y_{s}\right)\right] \cdot\left[(P+\bar{Q})\left(Y_{1}, \cdots, Y_{s}\right)\right]=1 .
$$

Let $h$ be any assignment, then by 5.1 we see that

$$
\left[(\bar{P}+Q)\left(h\left(Y_{1}\right), \cdots, h\left(Y_{s}\right)\right)\right] \cdot\left[(P+\bar{Q})\left(h\left(Y_{1}\right), \cdots, h\left(Y_{s}\right)\right)\right]=1 .
$$

By $1.2(\mathrm{vi})$, this means that

$$
(\bar{P}+Q)\left(h\left(Y_{1}\right), \cdots, h\left(Y_{s}\right)\right)=1
$$

and

$$
(P+\bar{Q})\left(h\left(Y_{1}\right), \cdots, h\left(Y_{s}\right)\right)=1 .
$$

(1) and (2) lead to

$$
P\left(h\left(Y_{1}\right), \cdots, h\left(Y_{8}\right)\right) \leqq Q\left(h\left(Y_{1}\right), \cdots, h\left(Y_{8}\right)\right)
$$

and

$$
Q\left(h\left(Y_{1}\right), \cdots, h\left(Y_{s}\right)\right) \leqq P\left(h\left(Y_{1}\right), \cdots, h\left(Y_{8}\right)\right),
$$

which give the desired conclusion.

We now give an interesting theorem which connects algebra with logic.

Theorem 5.3. In order that the $\boldsymbol{\aleph}_{0}$-valued logic be complete it is necessary and sufficient that the $M V$-algebra $L$ be representable.

Proof. (Necessity). By 4.9 it is sufficient to prove that every element 
$x \neq 0$ in $L$ is not included in some maximal ideal. Let an element $x$ be given with a representation $x=P\left(Y_{1}, \cdots, Y_{s}\right)$. Since $x \neq 0$, by 5.1 (i.e., our assumption) there exists an assignment $h$ for which

$$
P\left(h\left(Y_{1}\right), \cdots, h\left(Y_{8}\right)\right) \neq 0 .
$$

We now extend the assignment $h$ to the set of all generators by arbitrarily assigning the value 0 to every generator not among the set $\left\{Y_{1}, \cdots, Y_{s}\right\}$. We define a set $M$ of elements of $L$ as follows: $M$ is the set of all elements $y$ of $L$ such that with respect to some representation $y=Q\left(Z_{1}, \cdots, Z_{p}\right)$, $Q\left(h\left(Z_{1}\right), \cdots, h\left(Z_{p}\right)\right)=0$. It is evident that $M$ is an ideal of $L$. Let $z \notin M$, this means that in some representation $z=R\left(W_{1}, \cdots, W_{q}\right)$ where $R\left(h\left(W_{1}\right), \cdots, h\left(W_{q}\right)\right) \neq 0$. Since the algebra $S(\infty)$ is locally finite, there exists an $n$ for which

$$
n \cdot R\left(h\left(W_{1}\right), \cdots, h\left(W_{q}\right)\right)=1
$$

and

$$
[\bar{R}]^{n}\left(h\left(W_{1}\right), \cdots, h\left(W_{q}\right)\right)=0 .
$$

Thus the element $\bar{z}^{n}$ with the representation $\bar{z}^{n}=[\bar{R}]^{n}\left(W_{1}, \cdots, W_{q}\right)$ belongs to $M$. By $4.7, M$ is a maximal ideal. By 5.2 , (1), and the definition of $M$, $x \in M$. Hence the necessity has been proved.

(Sufficiency.) First of all, by the definition of $L$, there is no difficulty in showing that if $P\left(Y_{1}, \cdots, Y_{s}\right)=1$, then $P\left(Y_{1}, \cdots, Y_{s}\right)$ is valid. Let us now assume that an element $x=P\left(Y_{1}, \cdots, Y_{s}\right)$ of $L$ is valid. We prove that $x=1$ by contradiction. Assume that $x \neq 1$, then, by 4.9 , there exists a maximal ideal $M$ such that $\bar{x} \notin M$. This means that $x / M \neq 1 / M$. Let us now consider the algebra $L / M$ which, by 4.7 , is locally finite. There are two cases:

CASE 1. $L / M$ contains an atom. In this case, by $3.19, L / M$ is isomorphic to $S(m)$ for some $m$. Let the isomorphism be $g$. Clearly the mapping $h(x)$ $=g(x / M)$ is an assignment of $Y_{1}, \cdots, Y_{s}$, and, furthermore, $h$ is a homomorphism of $L$ onto $S(m)$. Since $x / M \neq 1 / M$, we see that

$$
1 \neq h(x)=h\left(P\left(Y_{1}, \cdots, Y_{s}\right)\right)=P\left(h\left(Y_{1}\right), \cdots, h\left(Y_{s}\right)\right) .
$$

This is a contradiction.

CASE 2. $L / M$ contains no atoms. In this case, since $x / M$ $=P\left(Y_{1} / M, \cdots, Y_{\delta} / M\right) \neq 1$, we see by 3.26 that, for a suitable choice of the element $x$ (of 3.26), there exists an element $y \in L / M$, an integer $m$ such that $\operatorname{ord}(y)=m$, and a sequence of integers $n_{1}, \cdots, n_{s}$ such that

$$
P^{\prime}\left(n_{1} \cdot y, \cdots, n_{s} \cdot y\right) \neq 1 \text {. }
$$

On considering the definition of the polynomial $P^{\prime}$, we see that the formula in (2) simply means that, in the algebra $S(m)$,

$$
P\left(n_{1} / m, \cdots, n_{s} / m\right) \neq 1 \text {. }
$$


It now follows from (3) that the mapping $h$ defined by $h\left(Y_{i}\right)=n_{i} / m$ for $1 \leqq i \leqq s$ is an assignment for which $P\left(h\left(Y_{1}\right), \cdots, h\left(Y_{s}\right)\right) \neq 1$. This is also a contradiction.

Since we have arrived at a contradiction in both cases, we see that the sufficiency is proved.

5.3 provides the algebraic method of which we spoke and by which we hoped to find an algebraic proof of the completeness of $\boldsymbol{\aleph}_{0}$-valued logic. Unfortunately, we see at present no simple and direct proof that $L$ is representable. The essential difference then between the two-valued case and the $\boldsymbol{\aleph}_{0}$ valued case is that not every $\mathrm{MV}$-algebra is automatically representable while every Boolean algebra is representable. We offer an algebraic proof of the following theorem which states, in the notation of [5], that if $P$ is valid then $\vdash B P P$. This, of course, is a much weaker result than the completeness; however, the proof we offer is very different in spirit from the proof in [5].

Theorem 5.4. If $x=P\left(Y_{1}, \cdots, Y_{s}\right) \in L$ and $P\left(Y_{1}, \cdots, Y_{s}\right)$ is valid, then $x+x=1$.

Proof. By contradiction. Suppose that $x+x \neq 1$, then by $3.9,4.4$, and 4.6 there exists a maximal ideal $M$ such that $x \cdot x \in M$. This clearly means that $\bar{x} \notin M$. Now the argument follows exactly as in the proof of the sufficiency of 5.3.

We conclude this section by considering some consequences when the axiom

Ax. 12(m).

$$
m \cdot x=(m+1) \cdot x
$$

(where $m$ is some fixed positive integer) is added to our original axioms Ax. 1-Ax. 11. If we now let A.5 $(m)$ be the natural translation of Ax. 12(m) into the notation of [5] and define the algebra $L(m)$ accordingly, then we obtain the following theorem:

THEOREM 5.5. For every $m \geqq 1$ and for every element $x=P\left(Y_{1}, \cdots, Y_{s}\right)$ of $L(m)$, (i) $x=1$, if, and only if, (ii) for every $n, 1 \leqq n \leqq m$, and for every assignment $h$ of $Y_{1}, \cdots, Y_{s}$ into $S(n), P\left(h\left(Y_{1}\right), \cdots, h\left(Y_{s}\right)\right)=1$.

Proof. Again, there is no difficulty in proving (ii) from (i). Let us now assume (ii) and, as usual, assume that (i) does not hold, i.e., $x \neq 1$. This implies that $x^{m} \neq 1$. From our axiom Ax. $12(m)$, we see that $m \cdot \bar{x}+m \cdot \bar{x}$ $=m \cdot \bar{x}$. By 1.16 , we see that $x^{m}+x^{m}=x^{m}$ and ord $\left(x^{m}\right)=\infty$. By 4.4 and 4.6 , there exists a maximal ideal $M$ such that $x^{m} \in M$. Clearly, $\bar{x} \notin M$. This means that the element $x / M \neq 1 / M$. Let us now consider the algebra $L(m) / M$. If $y \notin M$, then by $4.7 \bar{y}^{n} \in M$ for some $n$. Due to Ax. $12(m)$, it is not difficult to see that the integer $n$ can always be taken so that $n \leqq m$. Thus, not only $L(m) / M$ is locally finite, but every element of $L(m) / M$ must have order $\leqq m$. By 3.20 , we see that $L(m) / M$ must contain an atom which is of order $\leqq m$. 
Now, 3.19 tells us that $L(m) / M$ is isomorphic to $S(n)$ for some $n \leqq m$. Let the isomorphism be $g$, and let $h(x)=g(x / M)$ for $x \in L(m)$. Then $h$ is an assignment of the elements $Y_{1}, \ldots, Y_{s}$ into $S(n)$ and, furthermore, $h$ is a homomorphism of $L(m)$ onto $S(n)$. Since $x / M \neq 1 / M$, we see that

$$
1 \neq h(x)=h\left(P\left(Y_{1}, \cdots, Y_{s}\right)\right)=P\left(h\left(Y_{1}\right), \cdots, h\left(Y_{s}\right)\right)
$$

which is a contradiction. Thus, the theorem is proved.

In terms of many-valued logics, 5.5 asserts that a formula $P$ is provable from the Axioms A. 1-A. 4 and A. $5(m)$ if, and only if, $P$ is valid in every $n$-valued logic for $n \leqq(m+1)$. By an easy analysis on the notions of validity for $\aleph_{0}$-valued and $n$-valued logics, we see that the following is true which we shall simply state without proof.

TheOREM 5.6. The completeness of the $\boldsymbol{\aleph}_{0}$-valued logic is also equivalent to the following: If $P\left(v_{1}, \cdots, v_{s}\right)$ is a polynomial function, then $P\left(Y_{1}, \cdots, Y_{s}\right)$ $=1$ in $L$ if and only if $P\left(Y_{1}, \cdots, Y_{s}\right)=1$ in each $L(m)$ with $1 \leqq m$.

\section{REFERENCES}

1. G. Birkhoff, Lattice theory, Rev. ed., 1948.

2. C. C. Chang, Proof of an axiom of Łukasiewicz, Trans. Amer. Math. Soc. vol. 87 (1958) pp. $55-56$.

3. J. Eukasiewicz and A. Tarski, Untersuchungen über den Aussagenkalkiil, Comptes Rendus des Séances de la Société des Sciences et des Lettres de Varsovie, Classe III, vol. 23 (1930) pp. 30-50.

4. C. A. Meredith, The dependence of an axiom of Łukasiewicz, Trans. Amer. Math. Soc. vol. 87 (1958) p. 54.

5. A. Rose and J. B. Rosser, Fragments of many valued statement calculi, Trans. Amer. Math. Soc. vol. 87 (1958) pp. 1-53.

6. M. H. Stone, The theory of representations for Boolean algebras, Trans. Amer. Math. Soc. vol. 40 (1936) pp. 37-111.

7. A. Tarski, Contributions to the theory of models I, Indag. Math. vol. 16 (1954) pp. 572581.

8. M. Wajsberg, Beiträge zum Metaaussagenkalk ül I, Monatshefte für Mathematik und Physik vol. 42 (1935) pp. 221-242.

Cornell University, ITHACA, N. Y.

University of Southern California, Los Angeles, Calif. 